\title{
Influencing Visual Judgment through Affective Priming
}

\author{
Lane Harrison \\ UNC-Charlotte \\ Charlotte, North Carolina \\ ltharri1@uncc.edu
}

\author{
Drew Skau \\ UNC-Charlotte \\ Charlotte, North Carolina \\ dwskau@uncc.edu
}

\author{
Steven Franconeri \\ Northwestern University \\ Evanston, Illinois \\ franconeri@northwestern.edu
}

\author{
Aidong Lu \\ UNC-Charlotte \\ Charlotte, North Carolina \\ aidong.lu@uncc.edu
}

\author{
Remco Chang \\ Tufts University \\ Medford, Massachusetts \\ remco@cs.tufts.edu
}

\begin{abstract}
Recent research suggests that individual personality differences can influence performance with visualizations. In addition to stable personality traits, research in psychology has found that temporary changes in affect (emotion) can also significantly impact performance during cognitive tasks. In this paper, we show that affective priming also influences user performance on visual judgment tasks through an experiment that combines affective priming with longstanding graphical perception experiments. Our results suggest that affective priming can influence accuracy in common graphical perception tasks. We discuss possible explanations for these findings, and describe how these findings can be applied to design visualizations that are less (or more) susceptible to error in common visualization contexts.
\end{abstract}

\section{Author Keywords}

Visualization; Affect; Emotion; Charts

\section{ACM Classification Keywords}

H.5.m. Information Interfaces and Presentation (e.g. HCI): Miscellaneous

\section{General Terms}

Human Factors; Design; Measurement.

\section{INTRODUCTION}

Visual reasoning involves many cognitive processes including memory, attention, learning, judgment, creativity, decisionmaking, and problem-solving [8, 11, 13, 22]. Yet research in psychology has found that short-term changes in emotion significantly influence several of these same cognitive processes $[18,29]$. While researchers have begun to identify key factors that influence cognition (e.g. emotion and personality

Permission to make digital or hard copies of all or part of this work for personal or classroom use is granted without fee provided that copies are not made or distributed for profit or commercial advantage and that copies bear this notice and the full citation on the first page. To copy otherwise, or republish, to post on servers or to redistribute to lists, requires prior specific permission and/or a fee.

CHI 2013, April 27-May 2, 2013, Paris, France.

Copyright 2013 ACM 978-1-4503-1899-0/13/04...\$15.00. traits), the impact that such factors have on a user's ability to perceive information remains largely unexplored.

Emotion, often referred to as affect in psychology, is typically defined by values along two dimensions: valence (positive or negative feelings) and arousal (the intensity of those feelings). To study the impact of affect on various cognitive tasks, many studies in psychology involve manipulating valence and/or arousal via emotional stimuli. This technique of inducing emotion in human participants is referred to as affective priming.

Affect has been shown to influence both low-level cognitive abilities (attention, memory) [1, 50] as well as high-level abilities (creativity, decision-making, problem-solving) [2, 6, 35]. Since recent research in visualization has identified a close relationship between a user's cognitive abilities with their ability to perceive and understand information in visualizations $[8,11]$, we hypothesize that there exists a relationship between affect and visual judgment.

In this paper, we describe an experiment which uses affective priming to influence visual judgment. Cleveland and McGill's seminal results [10] serve as a basis for this graphical perception study, as they have been replicated and extended by Heer and Bostock [24] as well as adapted to study the effect of social information on visual judgments by Hullman et al. [28]. Specifically, we follow Cleveland and McGill's experiments which compare user accuracy with various position-length chart types (see Figure 1). We also extend their work by building on both recent crowdsourced graphical perception studies [16, 20] and affective priming studies $[14,27]$ to examine how emotion influences visual judgment [23].

We conduct an experiment which combines affective priming techniques with visual judgment tasks using Amazon's Mechanical Turk (AMT hereafter). We initially performed two pilot studies to verify our study design, emotional stimuli, and emotion-measurement metrics. For our main experiment, we recruited 963 participants to be either negatively or positively primed and to complete several visual judgment tasks similar to those found in Cleveland and McGill [10], Heer and Bostock [24], and Hullman et al. [28]. Our results indicate that affective priming significantly influenced the visual 
judgments of participants, and that positive priming improved accuracy.

\section{Contributions}

This paper makes a number of contributions to our understanding of how emotion, a fundamental component of human cognition, relates to graphical perception, a fundamental component of visualization:

- First, we present experimental evidence demonstrating that affective priming can significantly influence visual judgment accuracy.

- Second, we corroborate these findings with related research in cognitive psychology and discuss how changes in affect arise in visualization contexts.

- Finally, we describe several cases in which these results can be used to help choose effective visualizations for different audiences and contexts.

To avoid confusion, we note a caveat about the use of the word perception in graphical perception studies. In these studies, participants must not only identify (perceive) the graphical elements (bars, pie slices), but also make judgments on the proportion of one element to another. Since these judgments involve attention, working memory, and mental translation/manipulation, they are cognitive in nature. One recent study uses the term "visual judgment", which we adopt here [28]. Throughout this paper, we refer to graphical perception studies which involve visual judgments.

\section{RELATED WORK}

\section{Graphical Perception}

Graphical perception is a fundamental component of visualization, and is the subject of several recent works [24, 28]. The results from these studies have been used extensively in both the development of automatic presentation systems [38] and as a basis for visualization design principles [5].

Recently, Heer and Bostock replicated and extended a portion of Cleveland and McGill's experiments [24] on various chart types using AMT (see Figure 1). The rankings obtained in their crowdsourced studies were similar to Cleveland and McGill's rankings. These results validate AMT as a viable, scalable platform for visualization research.

Hullman et al. also leverage AMT and revise Heer and Bostock's graphical perception experiment [28] to explore the impact of social information on visual judgment. Their results suggest that visual judgment can be significantly influenced by adding social information, which has implications in collaborative and social visualization environments. Similarly, we explore the effect of affective priming on graphical perception (instead of the effect of social information on graphical perception). Our experiment also includes additional chart types in order to explore possible cognitive mechanisms (e.g. attention, memory, embodied cognition) through which affect influences visual judgment.
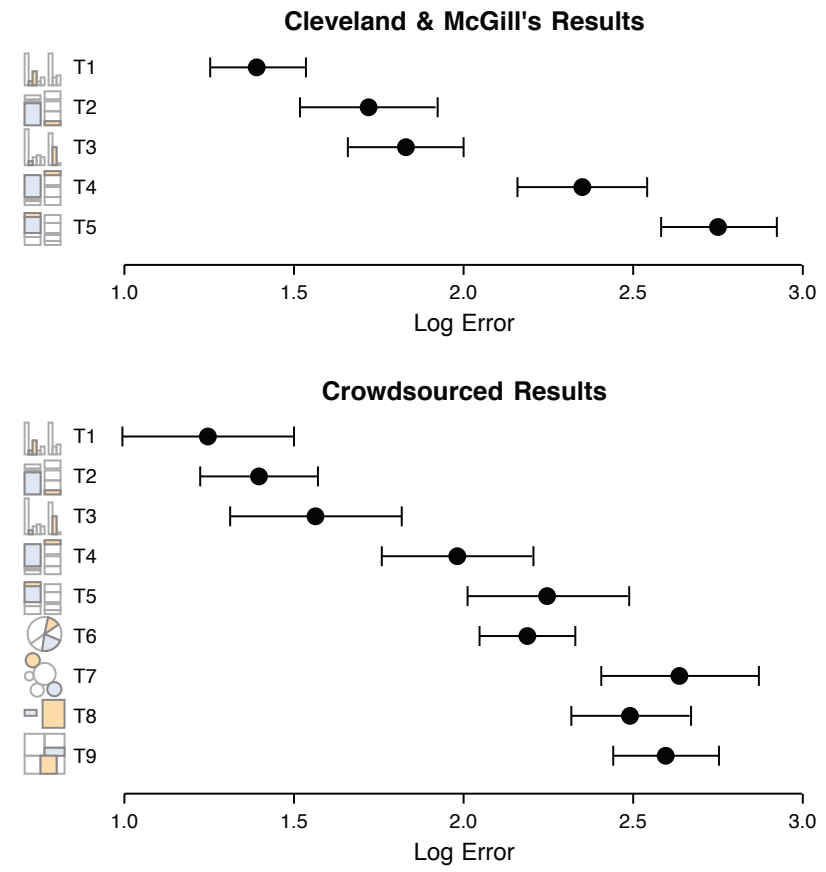

Figure 1. Cleveland and McGill's results and Heer and Bostock crowdsourced replication (from [24]).

\section{Affect and Cognition}

There is a large body of research on affect and its influence on various cognitive processes. These processes include attention, memory, creativity, problem-solving, decision-making, and others $[2,18,29,50,51]$. Early literature in this area focused on behavioral studies, but has recently been supplemented by studies which have identified the neurological factors related to emotion and cognition [51]. Here, we discuss research as it relates to the cognitive processes used in visualization. Specifically, we focus on attention and memory since they are low level components and, thus, are relevant to the low-level nature of graphical perception.

Several studies have explored the relationship between emotion and attention [40, 51], and specifically how attention relates to visual processes [50]. These studies show that when affective priming precedes cognitive tasks, the effect of the prime is carried over and influences attention regulation [40, 43]. Positive priming is usually linked to better performance [44], but there is also evidence that negative priming can also produce desirable behavior [19] such as increased caution in decisions involving risk. For a discussion on possible neurological factors and other attention regulation processes, see Vuilleumier and Huang [51].

In addition to impacting attention, emotion has been shown to play a role in memory [31]. Studies show that emotion can have a significant impact on long-term recall [2], as well as influence working memory $[1,32]$. Recent research also shows that negative affect can support memory of details, while positive affect increases working memory performance and supports memory of the "big picture" of situations [1, 32]. Another experiment found that anxiety (which falls under neg- 
ative affect) can significantly disrupt visuospatial working memory, leaving verbal working memory intact [45]. Our study is partially based on these prior findings, and apply the same text-based affective priming techniques as presented by Goeritz and Verheyen [21, 48].

Judgment (or decision-making) is defined as the process by which one assesses future outcomes and examines various alternatives, typically through cost-benefit analysis [3]. Emotion plays a significant component in decision-making, as each possible outcome is associated with emotional consequences $[3,37]$. In fact, emotion is so strongly linked to decision-making that persons who are perceptually and cognitively normal, except for an inability to experience emotions, are incapable of making even basic decisions [12]. Other studies have shown that affect also plays a role in planning and risk perception $[6,37]$.

Positive emotions have been shown to support creativity and open-ended reasoning [18]. A recent crowdsourced experiment showed that positive priming (using images) increased the quality of ideas generated on a range of tasks requiring creativity [35]. These results were used to make design suggestions for creativity-support software. Positive emotion is also linked to better performance in creative and general problem-solving [18, 29, 30].

In general, positive emotions tend to lead to better cognitive performance, and negative emotions (with some exceptions) lead to decreased performance. Yet we currently do not know to what extent affect can impact visualization use and performance. Hence the motivation for our study: to prime participants with positive or negative affect and to measure their performance on visual judgment tasks.

As indicated earlier, emotion is typically defined along two axes: valence and arousal. In psychology research, several methods have been developed for measuring valence (positive/negative feelings) and arousal (the strength of the feelings). Some of these include physiological sensors, facevideo processing, and neurological sensors [34, 42]. Physiological sensors excel at detecting arousal, but less so at valence [41]. The most widely used and validated method is the survey/self-report, especially for assessing immediate emotion (as opposed to, for instance, emotion experienced a week prior) [41]. The Self-Assessment Manikin (SAM) scale, shown in Figure 2, has been successfully used in crowdsourced experiments on affective priming and creativity in HCI by Lewis et al. [35]. Based on these results, we also use SAM in our experiments.

\section{STUDY DESIGN}

There are several key differences between existing techniques for research in affective priming and graphical perception that render their integration non-trivial. For example, affective priming studies are inherently between-subjects, since both the exact duration of a prime and the interactions between subsequent negative and positive primes is uncertain [25]. In contrast, graphical perception studies are typically within-subjects and require participants to interact with multiple charts, making participation time longer [24]. Given the
Select the figure (or between figures) that most closely corresponds to how you feel.

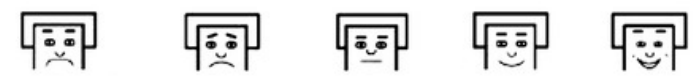

$0 \quad 0 \quad 0 \quad 0 \quad 0 \quad 0 \quad 0 \quad 0 \quad 0$

Select the figure (or between figures) that most closely corresponds to how you feel.

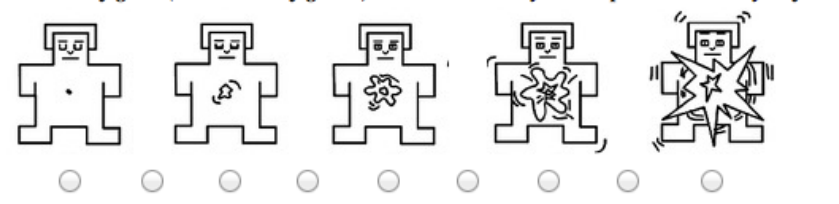

Figure 2. The 9-point Self-Assessment Manikin (SAM) scale for measuring valence (top) and arousal (bottom) [33].

intrinsic differences between these two types of studies, we discuss how we altered previous graphical perception experiment design to be more suitable for affective priming. Figure 3 shows an overview of the resulting study design and procedure.

\section{Charts}

Several previous studies on graphical perception included 10 datasets and allowed participants to work with all datasets and chart types. As our first task was to adapt a graphical perception study for affective priming, we found the length of previous studies to be a concern. The longer the study, the more risk of losing a successful prime [25]. To address this, we first reduced the number of randomly-generated test datasets from 10 to 5 . We ensured that the proportions of the marked elements that participants were to judge were similar to Cleveland and McGill's study, with ratios ranging from $13 \%$ to $85 \%$. Furthermore, we also restrict our participants to working with one chart type and priming condition, making our study a between-subjects design. Similar to traditional priming experiments, a between-subjects design is necessary since it ensures that no participants see the priming stimulus more than once and become accustomed to it.

In Heer and Bostock's study, some charts have 5 graphical elements ${ }^{1}$, such as pie charts, while some include 10 , such as bar charts, stacked bar charts, and treemaps. We sought to have even amounts of data elements in each chart. Therefore, all of our charts consisted of 5 graphical elements, with the exception of the stacked-bar chart. The stacked bar chart contains two columns, so including only 5 elements would create an uneven number of elements in each column (e.g. 3 in one bar and 2 in the other), possibly leading to confusion.

There are more length-based and rectangular charts in Heer and Bostock's study (shown in Figure 1). Also, the pie charts used in both Cleveland and McGill's as well as Heer and Bostock's study are unordered and do not start at 0 degrees, which may affect accuracy. To create a more even representation of length, angle, and area judgments, we reduce the number of stacked bar cases from three to one, and include an additional pie chart that is both ordered and starts at 0 degrees. We also include a bar chart with bars running horizontally instead of vertically, to test for a possible connection

\footnotetext{
${ }^{1}$ A graphical element is a component of a visualization. For example, a slice in a pie chart or a bar in a bar chart.
} 

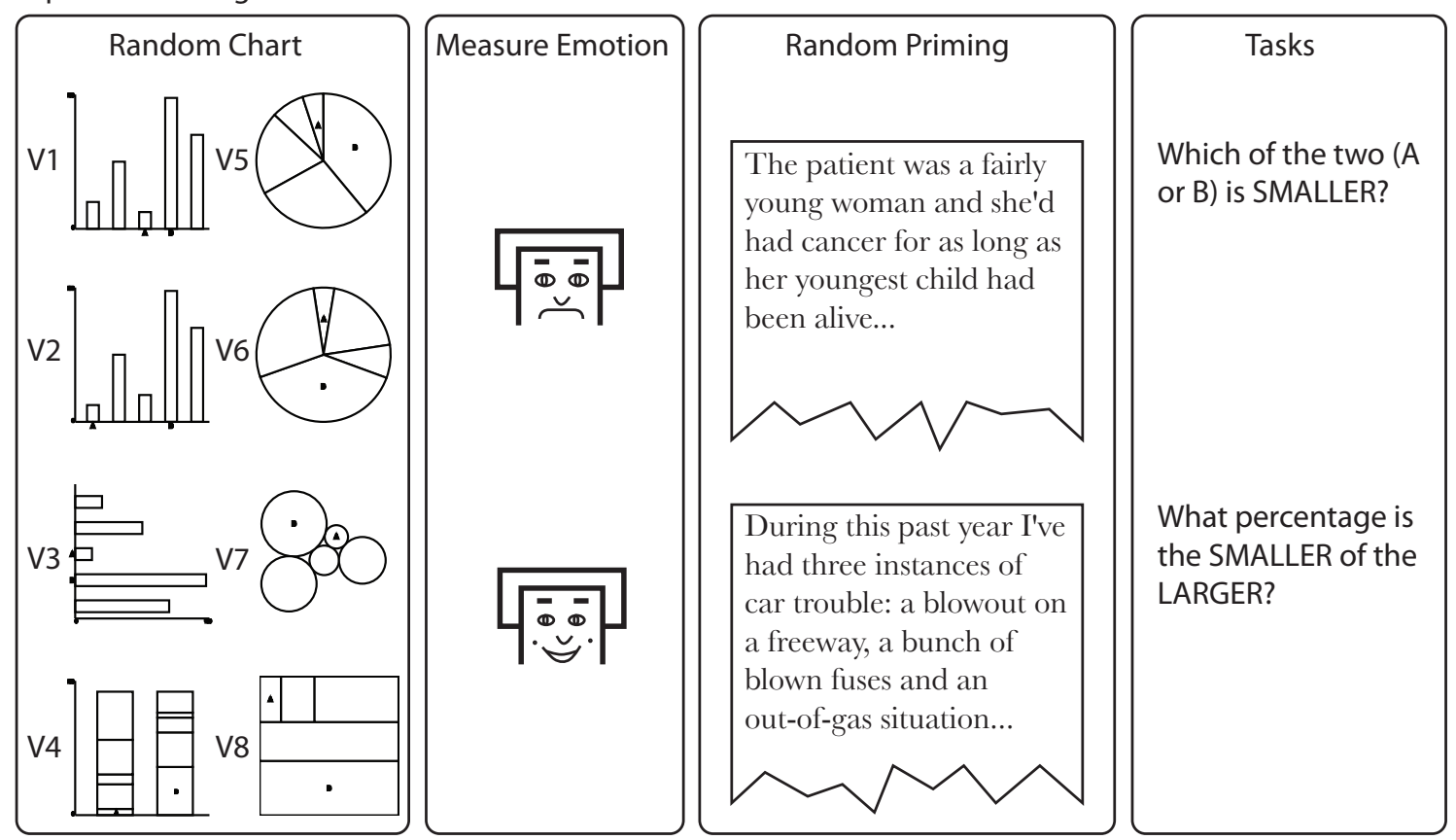

Measure Emotion

Output:

Pre-Valence

Pre-Arousal

Verification Question

Accuracy

Post-Valence

Post-Arousal

Figure 3. In our main experiment, participants were given either negative or positive text-based emotional-stimuli and completed visual judgment tasks with one of the eight chart types.
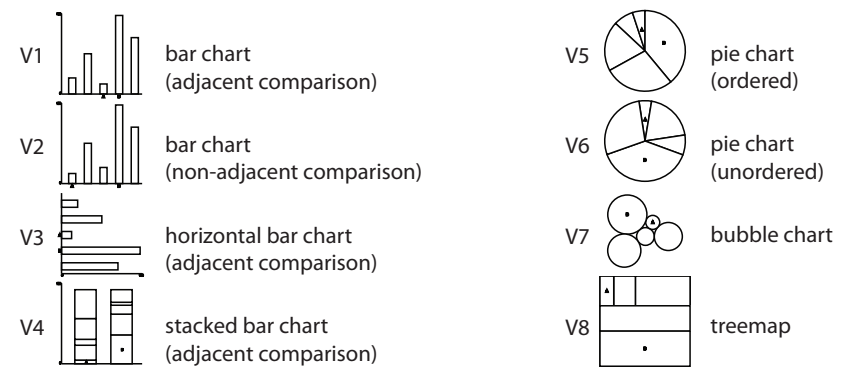

Figure 4. The charts used in our crowdsourced experiment.

between chart orientation and embodied cognition based on work by Ziemkiewicz and Kosara [53]. An example of each the 8 charts we use is shown in Figure 4.

\section{Affect Measurement}

We chose the Self-Assessment Manikin (SAM) scale for testing the valence and arousal of the participant. The SAM scale is widely used in psychology, in part because graphical depictions of emotion are cross-cultural [33, 35]. Unlike other surveys such as the Positive and Negative Affect Schedule (PANAS), SAM uses graphical depictions of emotions, which are both cross-cultural and take less time to complete [47], which makes SAM a widely used tool in psychology and HCI research, especially for studies that use the AMT platform, which often involves international participants [35, 39].
Figure 2 shows the images used in SAM. Selections can be made in between the figures, for a total of 9 possible choices. Our work is primarily concerned with the effect of valence. However, we also collect arousal scores to ensure that only valence is affected by the stimuli.

\section{Affect Stimuli}

Several methods have been introduced for priming participants with positive or negative affect. Common methods include images, text (stories), videos, sounds, word-association tasks, and combinations thereof. Such methods are commonly referred to as Mood-Induction Procedures (MIPs). We chose text-based stories as our MIPs for several reasons.

First, verbal memory is separate from visual memory [45]. This separation is important for visual judgment tasks because using text-based stimuli helps ensure that there is no interaction or interference between the priming section of the study and the visual judgment part. Another reason is that using stories makes it easier to hide the true nature of a study. Whereas graphic images are often an obvious cue of trying to elicit an emotional response, stories can be more subtle. Finally, we believe that emotional stimuli similar to our text-based MIPs are more likely to be encountered by users than the significantly more graphic images and videos used in other studies [21].

For our main experiment, we evaluated several short stories found in the New York Times website for their emotional con- 
tent. The stories were carefully selected to be relatively short (around 800 words) to ensure that participants could complete the study in a reasonable amount of time. Additionally, stories had to contain content that would be considered negative or positive by participants with divergent cultural/ethnic backgrounds. In the end, we chose two stories from the New York Times, one negative story describing hospice care, and one positive story about the kindness of strangers.

To validate that these stories elicit the desired affective response from the participants, we ran a pilot study on AMT with $n=40$ participants. To help ensure that the reported valence and arousal was not an effect of satisficing (i.e. participants trying to submit the answers they think the experimenters want), participants were only given the SAM as a post test in this pilot study. Satisficing was less of a concern in the full study because the stories were already determined (via the pilot) to induce the desired primes.

After accepting the task, participants read the story, with instructions to pay attention to the overall story more than the details. After the reading was complete (we made no time restrictions), they were asked a verification question (multiple choice) about the overall content of the story. The story was hidden during the verification questions. Next, they were given the SAM as a post-test. Finally, they were invited to share any additional comments about the story.

The results of the story validation showed a significant difference between the positive and negative groups for valence for both stories $(p<.05)$. We also checked for significant differences in arousal, since having one story that produced low arousal and one that produced high arousal could be a confounding factor in the experiment [1]. However, no significant difference was found between stories for arousal $(p>.05)$.

\section{PILOT}

In a pilot study, we tested text-based affective priming with Cleveland and McGill's position-angle experiment, which compared performance between bar charts and pie charts. The goal of this experiment was to determine whether to use raw valence scores or change in reported valence. Change in reported valence in the intended positive or negative direction is the same measure used in other affective priming studies [21, 48]. However, other studies have grouped participants by raw valence scores [35]. For instance, on our 9-point SAM scale, anyone below a 4 would be considered negatively primed, and anyone above a 7 would be considered positively primed. Alternately, if a participant reported a positive change in emotion, they would be in the positive group (and vice-versa).

After running the pilot and analyzing participants' performance, we found that performance on both charts was affected when the reported pre- and post-valence scores were changed in the intended direction (post $>$ pre for positive, and post $<$ pre for negative).

In this work, we call such a change as a "successful" prime. Based on this pilot, we formed the hypothesis that successful affective priming will significantly influence error in visual judgment across all chart types.

Below we will describe the experiment used to test this hypothesis. In general, we incorporate text-based affective priming techniques and follow Cleveland and McGill's study on graphical perception and Heer and Bostock's recreation of the study on AMT. In the following section, we discuss the changes we made to these studies to better suit them for affective priming.

\section{EXPERIMENT}

To validate our hypothesis that affect influences accuracy in graphical perception, we conducted a large-scale experiment using AMT. We chose to follow Heer and Bostock's crowdsourced replication of Cleveland and McGill's seminal study on graphical perception $[10,24]$, making necessary changes to effectively incorporate affective priming, described in the study design. We verified the effectiveness of our emotional stimuli and determined that change in valence should be examined through two pilot studies.

Amazon's Mechanical Turk (AMT) is a service that allows workers to complete various web-based tasks in exchange for micro payments. It has been used for several graphical perception studies $[24,28]$, as well as studies exploring other cognitive aspects of visualization [52]. Another recent work used AMT as a platform for exploring affective priming and creativity [35].

\section{Participants}

We recruited 963 participants through AMT. It took a approximately five days to gather all responses.

Our study used a total of eight charts with the two priming groups (positive/negative). This resulted in a total of 16 groups (V1-Positive, V1-Negative, V2-Positive, etc.). It is well-documented that positive priming is more difficult to obtain than negative [21]. Therefore, we assigned more participants to the positive groups, to increase the number of successfully positively primed participants. Participants were randomly assigned to a priming group (with a $1 / 3$ chance of being in the negative group, and $2 / 3$ chance of being in the positive group) and randomly assigned to a chart group (charts shown in Figure 3).

The task was advertised on AMT as involving reading a story and answering several short questions afterwards. The task description did not reveal that we would be measuring mood or working with charts. Although we used stories found in newspapers, participants were instructed that they could quit the task at any time if they found the material offensive. After completing the study, participants were initially paid $\$ 0.20$, which was later increased to $\$ 0.35$ to speed completion time.

\section{Procedure}

After a participant has been assigned to a chart type and priming group, the participant was first given the SAM scale as a pre-test to record their valence and arousal. Next, participants were given the positive or negative story to read with the instructions that "the overall story is more important than 


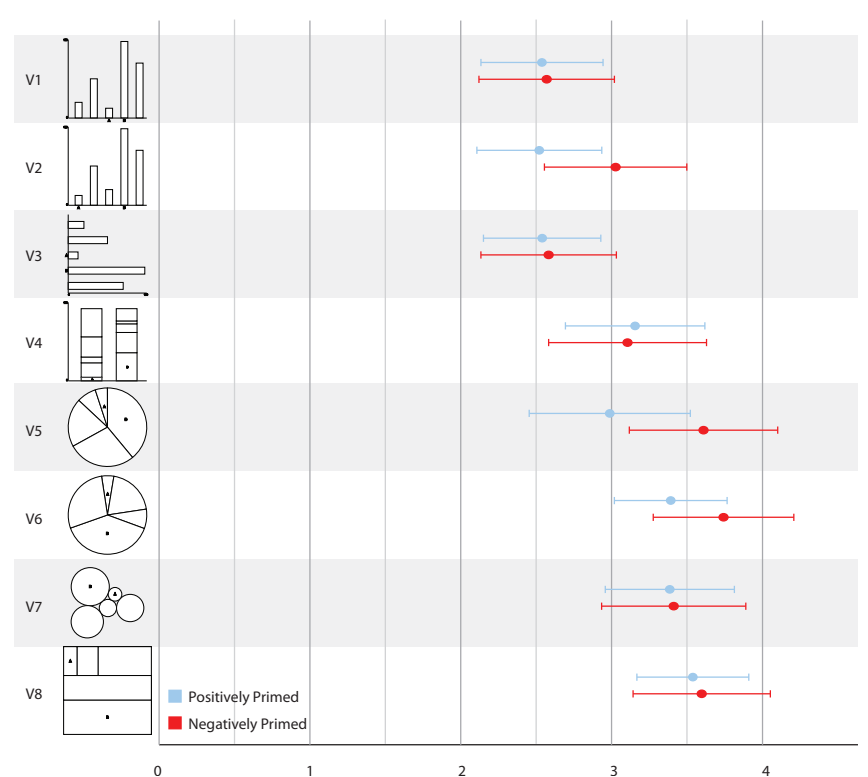

Figure 5. Results for all participants.

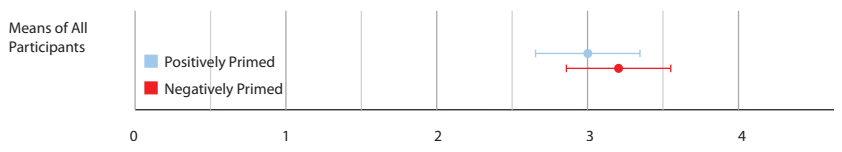

Figure 6. Total means for all participants.

the details". After reading was complete, participants were asked a simple verification question regarding the content of the story (the story was hidden at this point). Next, participants began the visual judgment tasks.

Each of the five tasks (all being the same chart type: bar, pie, or one of the others in Figure 4) were presented in random order. Recall that the datasets for each chart were generated in the same manner as Cleveland and McGill's and other graphical perception studies. Participants were instructed to make quick but accurate visual judgments, and to avoid using any outside tools. Similar to Heer and Bostock's study, participants were asked the following two questions about the charts: First, "Which of the two (A or B) is SMALLER?" followed by "What percentage is the SMALLER of the LARGER?”.

After the visual judgment tasks were completed, participants were given the SAM again as a post-test. The SAM was given after the judgment tasks to ensure that the prime lasted through the duration of the visual judgement tasks. Finally, they were invited to submit any additional comments about the task.

\section{Results}

Our study adhered to a between-subjects design, since participants were given a single chart and priming combination. We excluded participants who incorrectly answered the story verification question, as well as those who either failed to answer or put the same answer for multiple questions (e.g. putting $50 \%$ for each judgment). In total, 299 of the 963 participants

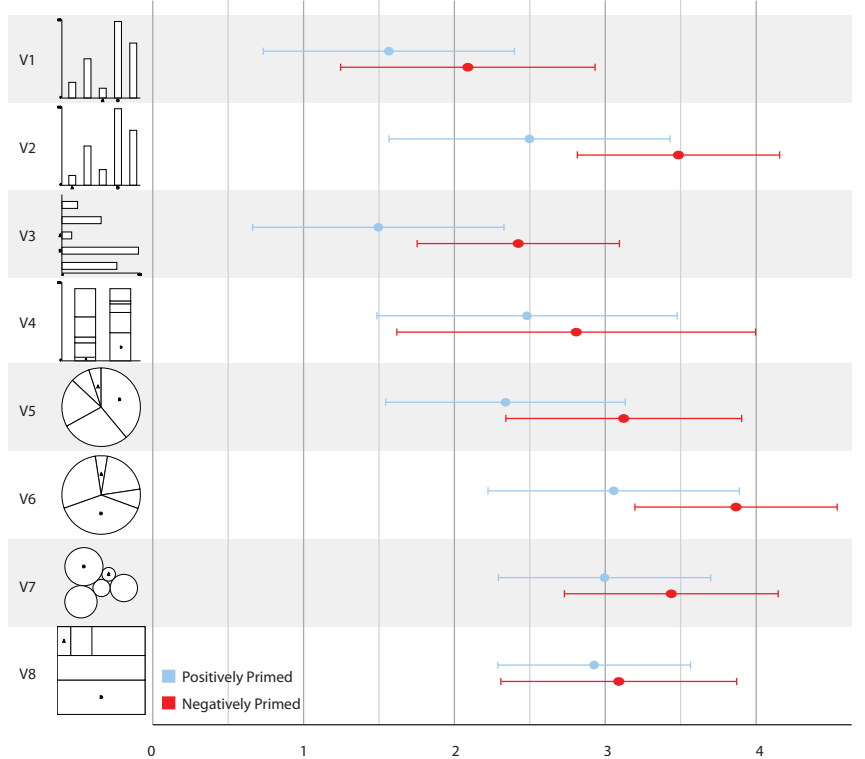

Figure 7. Results for successfully primed participants.

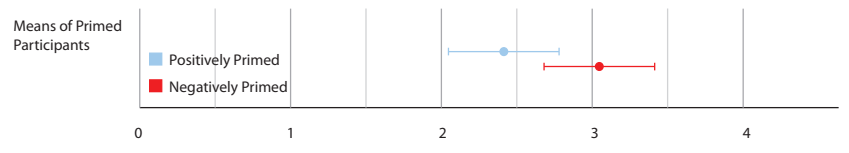

Figure 8. Total means for successfully primed participants.

were removed, which is consistent with prior findings of error rates in AMT studies (see Bernstein et al. [4]).

Following previous graphical perception studies of Cleveland and McGill, Heer and Bostock, and Hullman et al., we use the midmeans of log absolute errors (MLAEs) using the following formula:

$$
\log _{2}\left(\mid j u d g e d \_p e r c e n t-\text { true_percent } \mid+.125\right)
$$

Similarly, we also follow these studies in using bootstrapping to calculate the mean confidence intervals for each chart and priming combination. The resulting confidence intervals are shown in Figures 5 and 7.

We divide our analysis into two cases, the first including all participants in the negative and positive groups, and the other including only participants who were successfully primed in the intended direction. The implications of each of these cases will be explored further in the discussion.

\section{Case 1: All participants}

This case includes every participant: 359 in the positive group and 305 in the negative group. Each chart and priming group has an average of 41 participants. This analysis consists of 3320 individual judgments.

For each chart in the graphical perception section, two data items were collected: which chart was perceived as smaller, and a number representing the percentage the smaller was of the larger (between 0 and 100). For each of these judgments, 
we compute the MLAEs using the formula mentioned previously. We then compute the mean error and $95 \%$ confidence intervals for each chart and priming type.

The results of these calculations are shown in Figure 5. Following Hullman et al., we also take the results for all charts in each priming group together, including all chart error scores for each participant in the negative- and positiveprimed groups. A t-test on these groups was not significant $t(662)=1.8318, p=.067$, This is not surprising because the difference between positive $(\mathrm{M}=3.00, \mathrm{SD}=1.39)$ and negative $(\mathrm{M}=3.20, \mathrm{SD}=1.475)$ is only 0.2 , which is a small difference in error.

Recall that in our pilot study, we explored two common metrics for defining negative and positive groups. One approach divides groups based on raw valence scores, and the other on whether the change in pre- to post- valence scores occur in the intended direction [21, 48]. The results of the pilot study found a possible influence for change in valence on visual judgment. Based on these results, we include the change in valence metric in our second case.

\section{Case 2: Successfully primed participants}

In this case we include those who were succesfully primed in the intended negative or positive direction. Specifically, we include those whose post-valence is higher than pre-valence for the positive group, and vice-versa for the negative group. This resulted in 87 participants in the positive condition and 120 in the negative condition for a total of $n=197$. There was an average of 13 participants for a single chart and priming condition (out of 16 total possible combinations). Each participant made judgments on 5 charts. Therefore, this analysis consists of approximately 985 individual judgments.

As before, we compute the MLAEs for each judgment and combine these to produce a mean errors and confidence intervals for each chart and priming condition. The results of these calculations are shown in Figure 7.

We then compare positive error with negative error using a t-test. This yields a significant effect for error $t(205)=$ $3.1560, p=.0018$, with error in the negative group being higher than that of the positive group. The lowest error appeared in the positive group $(\mathrm{M}=2.42, \mathrm{SD}=1.47)$, meaning participants in the positive group performed better on visual judgment tasks than those in the negative group $(\mathrm{M}=3.05$, $\mathrm{SD}=1.49$ ). These results are consistent with our hypothesis that affective priming can significantly impact visual judgment performance in participants who report a change in valence scores.

An interesting finding is that it appears that positive priming tends to improve performance, rather than negative priming decreasing performance. We see this by comparing the data in Figures 6 and 8. It appears that, on average across all charts, negative priming stays about the same. To test this, we compare the successfully primed positive group (M $=2.42, \mathrm{SD}=1.47)$ against all positively primed participants $(\mathrm{M}=3.00, \mathrm{SD}=1.39)$. Doing so, we see a significant effect for error $t(444)=3.5634, p<.001$, with the successfullyprimed participants having significantly lower error than the total positive group. This supports the notion that positive priming can improve visual judgment accuracy.

Other interesting findings can be seen by looking at the individual difference in charts in the Figure 5. Following Cleveland and McGill as well as Heer and Bostock, rather than test each chart pair for significant differences, we will simply discuss the relative rankings between charts and the effects of affective priming in the following section.

\section{DISCUSSION}

Consistent with past research on the impact of affect and low level cognitive processes $[40,44]$, we found that affective priming also influences performance on visual judgment tasks involving several chart types commonly used in visualization [10, 24, 28]. Furthermore, as much research on affect has focused on the relationship between positive affect and increased cognitive performance [29, 30, 44], we also found that successful positive priming yielded significantly better visual judgment performance.

There are many ways in which the affect might impact performance. Given the low-level nature of the visual judgment tasks described in our experiment (the perception of larger/smaller elements and the estimation of the difference in their sizes) we find it useful to focus on low-level cognitive processes such as attention and working memory, which can be enhanced by priming positive affect [50, 1, 32].

While positive affect might improve these processes partly through higher overall engagement and alertness [44], it is also likely that different moods place observers in states that are better or worse for given tasks. For instance, positive moods can expand the scope of the conceptual 'spotlight', in creativity tasks where observers must generate new uses for tools [35], identify words that form compounds with an arbitrary set of other words [44], or solutions to anagrams [46].

But positive moods can also expand the scope of the perceptual spotlight of attention [15]. They encourage an observer to process a larger spatial area of the world in a single glance $[44,20]$, relative to negative or anxious moods that constrict this spatial area [16]. These effects are often demonstrated by asking observers to make a decision about a small spatial area (e.g. discriminate a letter at the center of a screen), and showing that incompatible information within the broader spatial area (e.g., another letter that tempts the 'wrong' answer) interferes more after positive mood priming than after negative or anxious mood priming.

Work from the perceptual psychology literature also suggests that some visual operations are more efficient under a broad spatial scope. These include determining the distance between two objects [7], deciding whether two objects are visually similar [17], or extracting statistical summary information (about e.g., size or orientation) from a collection of objects [9]. In contrast, a more narrow spatial scope may be necessary for processing spatial relations between objects $[7$, 17] or accessing fine details [40].

These differences in the perceptual scope of attention might serve as a mediating factor that causes mood to impact per- 
ceptual performance. A broad scope of attention might be beneficial for tasks where relevant information is more spatially distant, such as a bar chart with a non-adjacent comparison (v2). Unordered pie charts (v5, v6), which are positively impacted even in the experiment case with all participants (Figure 5), may similarly benefit from larger perceptual scope.

Mood may also impact perceptual performance via altering the performance of visual working memory. Visual working memory capacity may be highly influenced by the scope of information that an observer attempts to encode at once. Attempting to encode a large number of objects at once may lead to lossy encoding of each item, in contrast to attempts to encode a smaller number of objects at once with more precise encodings [49]. Changing mood might change perceptual scope, which could in turn alter the balance between the capacity and level of detail of visual memories for previously seen information. Depending on the visual task, information about more objects (but lossier) or information about fewer objects (but more detailed) might be more beneficial to observer performance.

Finally, in examining Figure 7, we can consider the trend where some charts appear to be less impacted by the affective primes - specifically treemaps (v8), bubble charts (v7), and stacked bar charts (v4). One possible reason for this stability effect is that the difficulty of these charts increases cognitive effort, which Hullman et al. suggests may lead participants to think about their decisions more carefully [27]. Specifically, both the treemaps and the bubble charts do not have axes, which Heer and Bostock discuss as a possible difficulty for users [24]. Also, the stacked bar charts used more graphical elements than the other charts, which Hollands and Spence found to increase cognitive effort [26].

\section{Comparison to Previous Graphical Perception Studies}

Our results preserve the general ranking from Heer and Bostock's study. However, equivalent charts in Heer and Bostock's study have lower error, despite the fact that we mimic their chart types and data-generation methods closely. We have identified possible reasons for this global increase in error, which can inform future work involving priming and graphical perception.

First, our tasks were longer than previous studies. In Heer and Bostock study as well as Hullman et al., each chart was given as an individual task. This means users could complete each tasks at will, rather than as part of one task. In contrast, due to our use of affective priming, our stories and charts had to be given together in one task. Additionally, Heer and Bostock as well as Hullman et al. make use of qualification tasks to verify and train participants for the chart-perception tasks. Qualification tasks serve two main functions: to ensure participants accurately follow instructions and to briefly train participants on the tasks. However, we omit a qualification since it would reveal the nature of the experiment and significantly extend the study length. Future experiments could explore how to effectively incorporate additional quality assurance methods into crowdsourced priming experiments, perhaps based on those discussed by Mason and Suri [39].
Learning effects could also account for better performance in Cleveland and McGill as well as Heer and Bostock. In Cleveland and McGill's experiment, each participant was given 50 tasks (10 charts and 5 datasets) as opposed to our 5, so some amount of learning is possible. Heer and Bostock also used a training task, the purpose of which is to help participants learn how to work with each chart type. This may have impacted the participant's abilities in performing visual judgments.

\section{Comments on Affective Priming Studies}

It is worth noting that the stories we used as emotional primes came from everyday newspapers. We followed this restriction in order to create a prime that is more common in everyday life. Similarly, we did not pursue images as a priming technique, since images that tend to produce strong changes in valence are both less common and tend to cause a significant change in arousal, which can be a confounding factor in affective priming studies [51]. Based on the results of this study, which has shown that common affective primes can significantly impact visual judgment, we believe that alternate priming techniques could be evaluated in future work as a means to further explore the relationship between emotion, cognition, and visualization.

In our pilot study, we found that although it is easier to prime people with negative affect, more benefits are gained from positive priming. However, because the average pre-valence across both groups was between 6 and 7, it was more difficult to obtain a positive delta in valence. This finding is corroborated by previous studies which have noted that most people begin in a slightly positive state, and that more even results can be obtained by assigning more participants to the positive priming group [48].

\section{IMPLICATIONS}

We have found that affect influences visual judgment, and that positive priming increases visual judgment performance. Based on these results, we discuss some possible implications regarding visualization design and evaluation.

One specific design implication comes from our finding that non-adjacent comparisons appear to be both less accurate and less influenced by affective priming in both the all-participants and successfully-primed participants case. Therefore, when designing interactive visualizations where emotion can be a factor, designers should ensure the user should be able to interactively change a non-adjacent comparison situation to an adjacent comparison situation or to use other interactions or views to better support non-adjacent comparisons.

Other implications are more broad. For instance, as visualizations become more common in everyday life, care must be taken to ensure that information is communicated accurately for a variety of audiences and contexts. In general, we note that designers have no control over the possible primingeffects users experience before encountering a visualization. It is possible that a user could be primed positively or negatively through a variety of means, such as stories read in a newspaper or interactions with coworkers. Since the results of our study suggest that positive priming improves visual 
judgment accuracy, future research could explore how to integrate positive priming into design.

On the other hand, visualization tool design often includes knowledge about the environments in which users interact with the system. For instance, a user in a disaster-response setting may be more subject to negative priming, whereas a user in gaming may be more subject to positive priming. Such information can be used to assess the probability of negative or positive emotions and their subsequent impact on cognitive processes. One approach designers could follow is that of Lewis et al. [35], who suggest that embedded images and other interface design elements could help manage affect in software systems.

Another possible situation is where strongly-emotional content is unavoidable, such as visualizations dealing with data that has strong potential for unintentional affective priming. For example, a study by Elting et al. [14] compared visualization formats for communicating clinical trial risk. In clinical trials, a significant difference in error can often mean a number of lives lost (or saved). Other common high-stress areas where visualization has been used include criminal investigation, finance (high risk), and disease control. Given the complex nature of the decisions made in these situations, we believe that it is necessary to investigate the relationship between visualization, emotion, higher-level cognitive processes.

\section{LIMITATIONS}

While positive priming is generally associated with better cognitive performance, negative priming can also impact higher-level cognitive processes. An example a recent study by Livingston et al. which found that negative content had more impact than positive content on a user's subsequent review of a game [36]. While positive priming can be used to positively influence perceptual accuracy, it is possible that the residual effects of positive emotions may negatively influence a higher-level task.

\section{CONCLUSION}

In this paper, we describe a crowdsourced experiment in which affective priming is used to influence low-level visual judgment performance. Our results suggest that affective priming significantly influences visual judgments, and that positive priming increases performance. We hope these findings serve as a step forward in better understanding the cognitive aspects related to visualization.

\section{ACKNOWLEDGEMENTS}

We thank the National Science Foundation (grant no. IIS1218170) for support of this research. Any opinions, findings, conclusions, or recommendations expressed in this paper are those of the authors and do not necessarily reflect the views of the National Science Foundation.

\section{REFERENCES}

1. Ashby, F. G., Valentin, V., and Turken, U. The effects of positive affect and arousal on working memory and executive attention. 2002, 245-282.

2. Bechara, A. The role of emotion in decision-making: Evidence from neurological patients with orbitofrontal damage. Brain and Cognition 55,1 (2004), 30-40.
3. Bechara, A., Damasio, H., and Damasio, A. R. Emotion, decision making and the orbitofrontal cortex. Cerebral Cortex 10, 3 (2000), 295-307.

4. Bernstein, M. S., Little, G., Miller, R. C., Hartmann, B., Ackerman, M. S., Karger, D. R., Crowell, D., and Panovich, K. Soylent: A Word Processor with a Crowd Inside. ACM CHI (2010), 313-322.

5. Bertin, J. Semiologie Graphique. Gauthier-Villars, 1967.

6. Bohm, G., and Pfister, H.-R. Anticipated and experienced emotions in environmental risk perception. Judgement and Decision Making 3 (2008), 73-86

7. Chabris, C. F., and Kosslyn, S. M. How do the cerebral hemispheres contribute to encoding spatial relations? Current Directions in Psychological Science 7, 1 (1998), 8-14.

8. Chen, C., and Czerwinski, M. Spatial ability and visual navigation: An empirical study. New Review of Hypermedia and Multimedia 3, 1 (1997), 67-89.

9. Chong, S., and Treisman, A. Attentional spread in the statistical processing of visual displays. Attention, Perception, \& Psychophysics 67, 1 (2005), 1-13.

10. Cleveland, W. S., and McGill, R. Graphical perception: Theory, experimentation, and application to the development of graphical methods. Journal of the American Statistical Association 79, 387 (1984), 531-554.

11. Conati, C., and Maclaren, H. Exploring the role of individual differences in information visualization. Advanced Visual Interfaces (AVI) (2008), 199-206.

12. Damasio, A. Descartes Error. New York: Grosset/Putnam, 1994.

13. Dou, W., Ziemkiewicz, C., Harrison, L., Jeong, D., Ryan, R., Ribarsky, W., Wang, X., and Chang, R. Comparing different levels of interaction constraints for deriving visual problem isomorphs. IEEE VAST (2010), 194-202.

14. Elting, L., Martin, C., Cantor, S., and Rubenstein, E. Influence of data display formats on physician investigators' decisions to stop clinical trials: prospective trial with repeated measures. British Medical Journal 318, 7197 (1999), 1527-1531.

15. Eriksen, C., and St. James, J. Visual attention within and around the field of focal attention: A zoom lens model. Attention, Perception, \& Psychophysics 40, 4 (1986), 225-240.

16. Eysenck, M., and Calvo, M. Anxiety and performance: The processing efficiency theory. Cognition \& Emotion 6, 6 (1992), 409-434.

17. Franconeri, S., Scimeca, J., Roth, J., Helseth, S., and Kahn, L. Flexible visual processing of spatial relationships. Cognition 122 (2012), 210-227.

18. Fredrickson, B. What good are positive emotions? Review of General Psychology 2, 3 (1998), 300-319.

19. Fredrickson, B., and Branigan, C. Positive emotions broaden the scope of attention and thought-action repertoires. Cognition and Emotion 19, 3 (2005), 313-332.

20. Gasper, K., and Clore, G. Attending to the big picture: Mood and global versus local processing of visual information. Psychological Science 13, 1 (2002), 34-40.

21. Goeritz, A. S. The induction of mood via the www. Motivation and Emotion 31, 1 (2007), 35-47.

22. Green, T., and Fisher, B. Towards the personal equation of interaction: The impact of personality factors on visual analytics interface interaction. IEEE VAST (2010), 203-210.

23. Harrison, L., Chang, R., and Lu, A. Exploring the impact of emotion on visual judgement. IEEE VAST (2012).

24. Heer, J., and Bostock, M. Crowdsourcing graphical perception: Using mechanical turk to assess visualization design. ACM CHI (2010), 203-212. 
25. Hermans, D., Houwer, J. D., and Eelen, P. A time course analysis of the affective priming effect. Cognition and Emotion 15, 2 (2001), 143-165.

26. Hollands, J., and Spence, I. Judging proportion with graphs: The summation model. Applied Cognitive Psychology 12, 2 (1998), 173-190.

27. Hullman, J., Adar, E., and Shah, P. Benefitting infovis with visual difficulties. IEEE TVCG 17, 12 (2011), 2213-2222.

28. Hullman, J., Adar, E., and Shah, P. The impact of social information on visual judgments. ACM CHI (2011), 1461-1470.

29. Isen, A. Positive affect facilitates creative problem solving. Journal of Personality and Social Psychology 52, 6 (1987), 1122-1131.

30. Isen, A. The influence of positive affect on clinical problem solving. Medical Decision Making 11, 3 (1991), 221-227.

31. Johnson-Laird, P., and Oatley, K. Cognitive and social construction in emotion. Handbook of Emotions, 2nd ed. (2000), 458.

32. Klein, K., and Boals, A. Expressive writing can increase working memory capacity. Journal of Experimental Psychology 130, 3 (2001), 520-533.

33. Lang, P., Bradley, M., and Cuthbert, B. International affective picture system: Affective ratings of pictures and instruction manual. NIMH Center for the Study of Emotion \& Attention (2008).

34. Larsen, R., and Fredrickson, B. Measurement issues in emotion research. 2003, 40-60.

35. Lewis, S., Dontcheva, M., and Gerber, E. Affective computational priming and creativity. ACM CHI (2011), 735-744.

36. Livingston, I., Nacke, L., and Mandryk, R. Influencing experience: the effects of reading game reviews on player experience. Entertainment Computing-ICEC 2011 (2011), 89-100.

37. Loewenstein, G., and Lerner, J. S. The role of affect in decison making. Oxford University Press, 2003, 619-642.

38. Mackinlay, J., Hanrahan, P., and Stolte, C. Show me: Automatic presentation for visual analysis. IEEE TVCG 13, 6 (2007), 1137-1144.

39. Mason, W., and Suri, S. Conducting behavioral research on amazons mechanical turk. Behavior Research Methods 44, 1 (2012), 1-23.

40. Phelps, E., and Carrasco, M. Emotion facilitates perception and potentiates the perceptual benefits of attention. Psychological Science 17, 4 (2006), 292-299.
41. Picard, R., and Daily, S. Evaluating affective interactions: Alternatives to asking what users feel. CHI Workshop on Evaluating Affective Interfaces: Innovative Approaches (2005).

42. Picard, R., and Scheirer, J. The galvactivator: A glove that senses and communicates skin conductivity. ACM CHI (2001), 1538-1542.

43. Pourtois, G., Thut, G., de Peralta, R. G., Michel, C., and Vuilleumier, P. Two electrophysiological stages of spatial orienting towards fearful faces: Early temporo-parietal activation preceding gain control in extrastriate visual cortex. Neuroimage 26, 1 (2005), 149-163.

44. Rowe, G., Hirsh, J., and Anderson, A. Positive affect increases the breadth of attentional selection. Proceedings of the National Academy of Sciences (PNAS) 104, 1 (2007), 383-388.

45. Shackman, A., Sarinopoulos, I., Maxwell, J., Pizzagalli, D., Lavric, A., and Davidson, R. Anxiety selectively disrupts visuospatial working memory. Emotion 6, 1 (2006), 40-61.

46. Swinyard, W. The effects of mood, involvement, and quality of store experience on shopping intentions. Journal of Consumer Research (1993), 271-280.

47. Thompson, E. R. Development and validation of an internationally reliable short-form of the positive and negative affect schedule (panas). Journal of Cross-Cultural Psychology 38, 2 (2007), 227-242.

48. Verheyen, C., and Goeritz, A. Plain texts as an online mood-induction procedure. Social Psychology 40, 1 (2009), 6-15.

49. Vogel, E., McCollough, A., and Machizawa, M. Neural measures reveal individual differences in controlling access to working memory. Nature 438, 7067 (2005), 500-503.

50. Vuilleumier, P., and Driver, J. Modulation of visual processing by attention and emotion: Windows on causal interactions between human brain regions. Philosophical Transactions: Biological Sciences 362, 1481 (2007), 837-855.

51. Vuilleumier, P., and Huang, Y.-M. Emotional attention: Uncovering the mechanisms of affective biases in perception. Current Directions in Psychological Science 18, 3 (2009), 148-152.

52. Ziemkiewicz, C., Crouser, R. J., Su, S., Yauilla, A. R., Ribarsky, W., and Chang, R. How locus of control influences compatibility with visualization style. IEEE VAST (2011), 81-90.

53. Ziemkiewicz, C., and Kosara, R. Preconceptions and Individual Differences in Understanding Visual Metaphors. Computer Graphics Forum 28, 3 (2009), 911-918. 